\title{
Downregulation of LANCL1-AS1 Promotes Tumorigenesis in Lung Adenocarcinoma Via the Modulation of miR-6748a-3p/PRSS8 Axis
}

\section{Guangjie Nie}

Shunde Hospital of Southern Medical University

Jun Liu

The First Affiliated Hospital of Guangzhou Medical University

Jiakang Liang

Shunde Hospital of Southern Medical University

Yi Yuan ( $\nabla$ yi1yuan8@163.com)

Shunde Hospital of Southern Medical University https://orcid.org/0000-0002-5547-3505

Research article

Keywords: lung adenocarcinoma, LANCL1-AS1, miR-6748a-3p, PRSS8

Posted Date: April 5th, 2021

DOI: https://doi.org/10.21203/rs.3.rs-386987/v1

License: (c) (i) This work is licensed under a Creative Commons Attribution 4.0 International License.

Read Full License 


\section{Abstract}

Background: Lung adenocarcinoma (LUAD) is one of the deadliest types of cancer worldwide. Previous studies have reported that the expression of LANCL1-AS1 was decreased in LUAD. Hence, the current study was conducted to confirm these findings and explore the probable mechanism of action.

Methods: LUAD and adjacent tissue samples were collected after the surgical procedure. For experiments in vitro, CCK-8 assay and colony formation assay were employed to explore the proliferation capacity while transwell assay was performed to explore the migration and invasion ability of LUAD cell lines. Luciferase reporter assay and RNA pull-down assay were used to confirm the interaction between LANCL1-AS1 and corresponding miRNA as well as the corresponding protein target of the miRNA predicted by online bioinformatics tools. The effect of different interventions on RNA and protein expression was confirmed by qRT-PCR and western blotting.

Results: It was observed that LANCL1-AS1 was low expressed in LUAD tissues and cell lines which was also associated with decreased survival of cancer patients. Overexpression of LANCL1-AS1 in cell lines was associated with decreased viability, proliferation, migration, and invasion, and with decreased subcutaneous tumor growth in nude mice. RNA pull-down and luciferase reporter gene assays suggested that LANCL1-AS1 interacted with miR-6748a-3p and LUAD tissue samples exhibited increased expression of miR-6748a-3p. Moreover, miR-6748-3p mimics decreased the RNA and protein expression of PRSS8 in LUAD cell lines. Interestingly, LUAD tissue samples exhibited low expression of PRSS8. The effects of LANCL1-AS1 overexpression on viability, proliferation, migration, and invasion capacity of LUAD cell lines were curtailed after the miR-6748a-3p overexpression or PRSS8 silencing.

Conclusion: Our results suggest that upregulation of LANCL1-AS1 can suppress tumor growth in LUAD through the modulation of miR-6748a-3p and PRSS8 dependent pathways.

\section{Background}

Lung cancer is the leading cause of cancer-related deaths and over a million deaths are attributed to lung cancer each year worldwide [1]. Despite the availability of new treatments, the 5-year survival of lung cancer is only about $12 \%$ to $15 \%$ [2]. LUAD is a subtype of non-small cell lung cancer (NSCLC) and the most common type of lung cancer in non-smokers and women $[3,4]$. LUAD has a strong association with previous smoking and may represent up to $40 \%$ of all lung cancers [2]. An early diagnosis of lung cancer remains the key to the treatment which necessitates finding new biomarkers and therapeutic targets.

Long-chain non-coding RNAs (IncRNA) are non-coding RNAs of about $200 \mathrm{nt}$ with diverse functions. One of its biological roles involves inhibition of the negative regulation of miRNA on downstream target genes through sponging miRNA [5]. The abnormal expression of IncRNA is often related to the occurrence and development of tumors [6]. For instance, IncRNA TUG1 has been shown to act as a tumor suppressor in human glioma [7]. IncRNA DGCR5 has been shown to exert inhibitory effects on papillary thyroid carcinoma via miR-2861 targeting [8]. Another IncRNA XIST has been reported to suppress prostate 
cancer by modulating the expression of RKIP through miR-23a [9]. The tumor suppressor effects of IncRNA-BCAT1 on colorectal cancer have also been reported [10]. LncRNAs can not only inhibit but also promote cancer. For example, IncRNA MIR4435-2HG promotes the development of lung cancer through $\beta$ -catenin signaling [11]. LncRNA RMRP has been suggested to act as an oncogene in lung cancer [12]. Another LncRNA CRNDE has been shown to promote cervical cancer through miR-183/CCNB1 axis [13]. The oncogenic role of LncRNA SNHG15 in prostate cancer has also been reported [14]. In a recent report, Wang et al. have reported that LANCL1-AS1 was down-regulated in LUAD based on machine learning and weighted gene co-expression network analysis [15]. Indeed, LANCL1-AS1 was proposed as a biomarker of LUAD in their report, but its role and mechanism in lung cancer have not been studied. LncBase online database predicts that has a miR-6748-3p binding site, and Targetscan online database predicts that miR-6748-3p targets PRSS8. Hence, in the current piece of investigation, we have explored the role of LANCL1-AS1 in LUAD and explored its possible mechanism of action involving the miR-6748-3p/ PRSS8 axis by using a variety of biochemical tools.

\section{Methods}

\section{Collection of clinical samples}

LUAD and normal tissue samples from adjacent tissues were collected from patients participating in the study at Shunde Hospital of Southern Medical University and immediately frozen at $-80^{\circ} \mathrm{C}$ until further use. Written informed consent was obtained from all the patient participants of the study before the surgical removal of the samples. All the procedures were approved by the ethical committee for human experimentation, Shunde Hospital of Southern Medical University (China), and were following the guidelines of the declaration of Helsinki.

\section{Animals and diets}

Nude mice of 5-6 weeks of age and weighing between $23 \mathrm{~g}$ to $25 \mathrm{~g}$ were purchased from Shunde Hospital of Southern Medical University, China, and kept under standard laboratory conditions (relative humidity $47 \pm 9 \%, 21 \pm 3{ }^{\circ} \mathrm{C}, 12 \mathrm{~h}: 12 \mathrm{~h}$ light-dark cycle) with food and water ad libitum. The mice were acclimatized for at least one week before experimentation. For the subcutaneous injection of LUAD tumor in nude mice, $2 \times 10^{6}$ cells with or without LANCL1-AS1 overexpression were suspended in $25 \mu \mathrm{L}$ of PBS and injected using a $27 \mathrm{G}$ needle [16]. The size of the tumor was measured after each week for a total period of 4 weeks. Tumor growth was expressed in terms of volume and weight and the tumor growth curve was constructed as a function of time. In accordance with the principles of animal welfare,the mice were euthanized by sodium pentobarbital injection, and the drug was injected intraperitoneally at a dose of $150 \mathrm{mg} / \mathrm{kg}$. All the experimental procedures were approved by the animal ethical committee of Shunde Hospital of Southern Medical University and in strict accordance with the international guidelines for animal experimentation.

\section{Cell culture and transfection}


$\mathrm{BEGM}^{\mathrm{TM}}$ bronchial epithelial cell growth medium (Lonza, Switzerland) was used for the culture of BEAS2B cells. H1650, PC9, H1975 cells were cultured in RPMI-1640 medium (Thermo Fisher Scientific, USA), while A549 cells were cultured in ATCC-formulated F-12K medium. Besides, 10\% fetal bovine serum (FBS) and $1 \%$ penicillin-streptomycin (Thermo Fisher Scientific, USA) was added to all the culture media. Standard laboratory conditions i.e. $5 \% \mathrm{CO}_{2}, 37^{\circ} \mathrm{C}$ were provided for the cell growth. For transfection of cells, Lipofectamine 2000 (Invitrogen, USA) was used according to the manufacturers' guidelines. Briefly, cells were seeded to be $70-90 \%$ confluent at transfection. Lipofectamine 2000 and sequence to be transfected were separately diluted in Opti-MEM ${ }^{\circledR}$ Medium 1:1, incubated for 5 minutes, and added to the cells. Transfected cells were kept at $37^{\circ} \mathrm{C}$ and analyzed after $48 \mathrm{~h}$.

\section{Quantitative Real-Time Polymerase Chain Reaction (qRT- PCR) analysis}

qRT-PCR assay was conducted according to the previously reported procedures [17]. TRIzol reagent (Thermo Fisher Scientific) was used for the extraction of RNA from the samples and RNA concentrations were determined by using a nano-drop spectrophotometer. The RNA was then converted to CDNA using DNA polymerase and M-MLV reverse transcriptase in a thermocycler (Bio-Rad, USA). Then, PCR was performed for 40 cycles of alternate temperatures of denaturation, annealing, and extension. SYBR Green PCR master mix (Thermo Fisher Scientific, USA) was used to analyze the gene expression using $2^{-\Delta \Delta C t}$ method. The melt-curve analysis was used for the analysis of the product specificity and all the experiments were repeated at least in triplicate. GAPDH and U6 are reference genes and used as internal controls. The sequences for primer used are as follows: LANCL1-AS1, F: AAAGGGGAAGAGGACAGGAA R: TTTTCAGGCTGTTTGCATTG; PRSS8, F: CTATGAAGGCGTCCATGTGTG, R: AGTTACACGTCTCACGACTGAT; miR-6748a-3p, F: AACAAGAGGACAGGGACAGAG R: CAGTGCGTGLUADGTGGAGT; si-PRSS8, F: ACAUUUUAAUCAUUUCUGCCC, R: GCAGAAAUGAUUAAAAUGUUU. GAPDH, F: ACGGGAAGCTCACTGGCATGG, R: GGTCCACCACCCTGTTGCTGTA; U6 F-5'-CTCGCTTCGGCAGCACA-3', R-5'AACGCTTCACGAATTTGCGT-3'.

\section{Western blotting}

For western blot assay, cells were lysed by using RIPA buffer (Proteintech, China) with added $1 \%$ protease inhibitor cocktail (Sigma, USA). The total protein content of the lysate was determined by the BCA method. The proteins were separated through SDS-PAGE and transferred onto polyvinylidenedifluoride (PVDF) membranes (Millipore, USA). The membranes were blocked by using a $4 \%$ solution of BSA for $2 \mathrm{~h}$ followed by incubation with primary antibody $(1: 500)$ overnight at $4{ }^{\circ} \mathrm{C}$ in dark. Then, the membranes were incubated with secondary antibody $(1 ; 1000)$ for $2 \mathrm{~h}$ at room temperature and protein bands were visualized by ECL assay kit (Bio-Rad, USA) and the band densities were compared using ImageJ software.

\section{Colony formation experiment}


This assay was conducted to analyze the proliferation ability of tumor cells after different interventions. For this purpose, cells in the logarithmic phase of growth were seeded onto 6 well plates and cultured for 14 days. After this period, the colonies started to appear which were washed twice with PBS followed by 20 min fixation with methanol. Crystal violet dye (Beyotime, China) was used to stain the colonies which were then air-dried and counted under a laboratory microscope (Olympus life science, Japan).

\section{Transwell assay}

Migration and invasion capacities of the tumor cells were analyzed by transwell assay [18]. For invasion assay, cells suspended in a medium without serum were placed in the upper chamber with a porous membrane that contained Matrigel solution (BD, USA). The lower chamber of the apparatus was immersed in a solution that contained a complete cell growth medium. After incubation for $24 \mathrm{~h}$ at $37^{\circ} \mathrm{C}$, cells at the upper side of the membrane were removed gently and cells retained at the lower side were fixed with $4 \%$ formaldehyde solution (Sigma-Aldrich, USA). The cells were then stained with $0.1 \%$ crystal violet solution (Sigma-Aldrich, USA) and counted under the lab microscope. The same protocol was followed for the migration analysis except that Matrigel was not used.

\section{Luciferase reporter gene assay}

For this assay, approximately $5 \times 10^{4}$ cells were seeded onto a 24 -well plate for $24 \mathrm{~h}$. Afterward, the transfection/transfection was carried out using Lipofectamine 2000 (Invitrogen, USA) at $37^{\circ} \mathrm{C}$ for $48 \mathrm{~h}$ according to the manufacturer's guidelines. Luciferase activity was performed using luciferase assay kit (Promega, USA) and the dual luciferase activity was measured by using microplate reader (Synergy H4 Hybrid Reader, BioTek, Winooski, USA) [17].

\section{RNA pull-down assay}

This assay was conducted according to the previously described procedures [19]. Biotin-labeled bioLANCL1-AS1 probe was provided by Sangon Biotech (Shanghai, China). Before the assay, cells were trypsinized and lysed and one of the lysate was kept as the input control. The other portion was incubated at $4{ }^{\circ} \mathrm{C}$ for overnight with magnetic Dynabeads M-280 Streptavidin beads (Invitrogen, Carlsbad, CA, USA) and the miR-6748-3p enrichment was determined by qRT-PCR analysis.

\section{Statistical analysis}

Statistical analysis of the results was conducted by using GraphPad Prism software V6. Kaplan-Meier survival curve was used to compare the survival rate of LUAD patients. Statistical analysis between two groups as done by Student's t-test while One-way ANOVA was used for the significance of the difference between more than two groups. $P$ values less than 0.05 were considered significant. The data represent the mean $\pm S D$ of three independent experiments.

\section{Results}




\section{LUAD tissues and cell lines have a decreased expression of LANCL1-AS1}

Web database TGCA analysis showed that LANCL1-AS1 expression was decreased in LUAD and was associated with decreased survival in cancer patients (Fig.1A). To further confirm these findings, the expression level of LANCL1-AS1 in 54 pairs of LUAD tissues and adjacent tissues was detected by qRTPCR. It was observed that LUAD tissues exhibited significantly decreased $(P<0.01)$ expression of LANCL1-AS1 compared with the controls (Fig.1B). In accordance, LUAD cell lines including A549, H1975, H1650, and PC9 exhibited significantly lower expression of LANCL1-AS1 than the normal lung epithelial cell line Beas-2B (Fig.1C). With the median expression value of LANCL1-AS1 in LUAD tissue in Fig.1B as a cut-off value, 54 LUAD patients were divided into high expression group $(n=27)$ and low expression group $(n=27)$, and Kaplan-Meier survival curve was used to evaluate the overall survival rate of the two groups. It was revealed that the low expression group of LANCL1-AS1 had significantly decreased $(P=0.0242)$ survival than the high expression group (Fig.1D). The relationship between the expression of LANCL1AS1 and clinical medical records of LUAD in the low-expression group and high-expression group was analyzed as shown in Table 1. The two groups were not significantly different in terms of age, gender, and tumor size (Table 1). It was observed that the high expression of LANCL1-AS1 was associated with a significantly higher incidence of poor tumor differentiation, lymph node metastasis, and higher TNM stage than low expression (Table 1).

\section{Overexpression of LANCL1-AS1 inhibited the proliferation, migration, and invasion of LUAD cells and increased the apoptosis rate}

Two LUAD cell lines i.e. A549 and H1975 with the lowest expression of LANCL1-AS1 in Fig.1B were selected and stable cell lines with LANCL1-AS1 overexpression (OE) were established (Fig.2A). It was observed that LANCL1-AS1 OE was associated with significantly reduced survival (Fig.2B), proliferation (Fig.2C) migration (Fig.2D), and invasion (Fig.2E) capacity of A549 and H1975 cells. Interestingly, subcutaneous injection of LANCL1-AS1 OE cells in nude mice resulted in significantly reduced subcutaneous tumor growth than control (Fig.2F).

\section{LANCL1-AS1 sponges miR-6748a-3p}

Online database starBase predicted the binding sites of miR-6748a-3p in LANCL1-AS1 (Fig.3A). To confirm these findings, two luciferase reporting experiments were carried out in A549 and $\mathrm{H} 1975$ cells. It was observed that compared with vector, overexpression of LANCL1-AS1 significantly decreased luciferase activity in A549 and $\mathrm{H} 1975$ cells, and the inhibition disappeared after the mutation of the LANCL1-AS1 binding site (Fig.3B). Besides, an RNA pull-down experiment was carried out with a biotinlabeled LANCL1-AS1 probe. Hence, it was observed that compared with the oligo probe, LANCL1-AS1 probe pulled down significantly more $(\mathrm{P}<0.01)$ miR-6748a-3p (Fig.3C). Moreover, overexpression of LANCL1-AS1 significantly reduced $(P<0.01)$ the expression of miR-6748a-3p than control which further confirms that miR-6748a-3p can directly interact with LANCL1-AS1 in A549 and H1975 cells (Fig.3D). Interestingly, LUAD tissue samples exhibited significantly increased $(P<0.05)$ expression of miR-6748-3p 
than the normal cells (Fig.3E), and an inverse correlation was observed between miR-6748-3p and LANCL1-AS1 expression in these tissues (Fig.3F), which further corroborates the in vitro findings.

\section{miR-6748a-3p targets PRSS8}

Web tool Targetscan database analysis predicted that PRSS8 has miR-6748a-3p binding sites (Fig.4A). Henceforth, dual luciferase reporting experiments were carried out in A549 and H1975 cells. It was observed that overexpression of miR-6748a-3p significantly inhibited $(P<0.01)$ luciferase activity in A549 and $\mathrm{H} 1975$ cells, and this inhibitory effect disappeared after the mutation in the PRSS8 binding site (Fig.4B). In addition, overexpression of miR-6748-3p resulted in decreased mRNA (Fig.4C) and protein (Fig.4D) expression of PRSS8 than control. Under the in vitro findings, LUAD tissue samples exhibited significantly reduced expression of PRSS8 than controls $(P<0.01)$ while PRSS8 and miR-6748a-3p expression exhibited a significant negative correlation $(R=0.8367, P<0.001)$ (Fig.4E, $F)$.

\section{LANCL1-AS1 suppresses LUAD progression by sponging miR-6748a-3p and thereby up-regulating PRSS8}

It was observed that A549 and H1975 LUAD cells that overexpressedLANCL1-AS1 also exhibited significantly increased mRNA (Fig.5A) and protein (Fig.5B) expression of PRSS8. In contrast, increased miR-6748 expression or si-PRSS8 significantly curtailed the mRNA and protein expression of PRSS8 in A549 and H1975 cells while overexpressing LANCL1-AS1 (Fig.5A, B). Interestingly, decreased viability (Fig.5C), colony formation (Fig.5D), migration (Fig.5E), and invasion (Fig.5F) capacities of LUAD cells caused by LANCL1-AS1 overexpression were partially rescued after increased miR-6748 expression or PRSS8 silencing in these cells. These observations suggest that LANCL1-AS1 exerts its modulatory effects on LUAD cells through miR-6748 and PRSS8 dependent pathways.

\section{Discussion}

Low expression of LANCL1-AS1 in LUAD tissue samples as well as in cell lines was observed during our study. The low expression of LANCL1-AS1 was strongly associated with poor prognosis and decreased survival in LUAD patients. Acha-Sagredo et al. have recently reported IncRNA dysregulation as a frequent event in NSCLC and their findings suggested that LANCL1-AS1 was down-regulated in NSCLC [20]. By using bioinformatics tool, Salavaty et al. have suggested that LANCL1-AS1 might be involved in the pathogenesis of LUAD and closely linked with overall survival [21]. Hence, our results are in corroboration of the previous reports and confirm their findings.

It was observed that overexpression of LANCL1-AS1 decreased the survival, proliferation, and metastasis capacity in LUAD cell lines by modulating PRSS8 through miR-6748a-3p sponging. The interaction between LANCL1-AS1 and miR-6748a-3p was confirmed by luciferase reporter gene assays and RNA pulldown assay. Interestingly, increased miR-6748-3p expression was also observed in LUAD tissue samples, and a significant negative correlation was noted between miR-6748-3p and PRSS8, which is in accordance with miR-6748a-3p targeting of PRSS8. Indeed, the IncRNA sponging of miRNAs has been reported in several previous reports and is a well-known mechanism involved during the action of InRNAs 
in cancer [22-24]. To our knowledge, no previous studies are available that discuss miR-6748a-3p and the current report presents the first evidence of the implication of miR-6748a-3p in any type of cancer. Nonetheless, several prior reports are available that have documented the tumor regulation by IncRNAs through miRNA sponging [25-29]. In contrast, the role of PRSS8 in the pathogenesis of cancer is well documented. For instance, Bao et al. have reported that PRSS8 suppressed the carcinogenesis and metastasis in colorectal cancer [30,31]. Loss of PRSS8 has shown to be closely associated with epithelial-mesenchymal transition in human bladder transitional cell carcinoma cell lines [32]. In contrast, increased expression of PRSS8 in cancer has also been reported in few studies. For instance, Tamir et al. have reported more than 100 fold increased expression of PRSS8 in ovarian cancer than normal or benign ovarian lesions and have suggested PRSS8 as a potential biomarker for the detection of ovarian cancer [33]. Interestingly, PRSS8 has been shown to inhibit the NSCLC tumor growth in both cell lines in vitro and in vivo which further strengthens the findings observed during the current report [34].

\section{Conclusions}

Reduced expression of LANCL1-AS1 promotes the tumor formation in LUAD and may serve as the biomarker and prognostic indicator of the disease. However, future studies are desirable in larger patient populations to further ascertain these findings.

\section{Abbreviations}

Lung adenocarcinoma (LUAD); non-small cell lung cancer (NSCLC); Long-chain non-coding RNAs (IncRNA); fetal bovine serum (FBS); Quanitative Real-Time Polymerase Chain Reaction (qRT- PCR); polyvinylidenedifluoride (PVDF); overexpression (OE).

\section{Declarations}

\section{Ethics approval and consent to participate}

In our research, LUAD and normal tissue samples from adjacent tissues were collected from patients participating in the study at Shunde Hospital of Southern Medical University. According to the Declaration of Helsinki, the INFORMED CONSENT was signed by all participating patients. All the procedures were approved by the ethical committee for human experimentation, Shunde Hospital of Southern Medical University (China), and were in accordance with the guidelines of the declaration of Helsinki.

\section{Consent for publication}

All authors consent to publication.

\section{Availability of data and materials}


All supporting data of this work, which are not available in public because of the ethical restrictions are available from the corresponding author upon request.

\section{Competing interests}

The authors report no conflicts of interest in this work.

\section{Funding}

There is no funding source for this work.

\section{Authors' contributions}

GN designed the project and collected data. JL analyzed the data and drafted the manuscript. JKL did almost all the experiments and were involved in data collection and analysis. YY conducted the methodology and administrated the project. All the authors revised and corrected the manuscript.

\section{Acknowledgments}

We thank the reviewers for their constructive comments.

\section{References}

1 Network CGAR. Comprehensive molecular profiling of lung adenocarcinoma. Nature 2014;511:543-50.

2 Myers DJ, Wallen JM. Cancer, lung adenocarcinoma. StatPearls [Internet] 2020.

3 Subramanian J, Govindan R. Lung cancer in never smokers: a review. J Nat/ Compr Cancer Netw 2019;17:xlv-liii.

4 Kenfield SA, Wei EK, Stampfer MJ, et al. Comparison of aspects of smoking among the four histological types of lung cancer. Tob Control 2008;17:198-204.

5 Militello G, Weirick T, John D, et al. Screening and validation of IncRNAs and circRNAs as miRNA sponges. Brief Bioinform 2017;18:780-8.

6 Loewen G, Jayawickramarajah J, Zhuo Y, et al. Functions of IncRNA HOTAIR in lung cancer. J Hematol Oncol 2014;7:90.

$7 \mathrm{Li}$ J, Zhang M, An G, et al. LncRNA TUG1 acts as a tumor suppressor in human glioma by promoting cell apoptosis. Exp Biol Med 2016;241:644-9.

8 Chen F, Yin S, Zhu J, et al. IncRNA DGCR5 acts as a tumor suppressor in papillary thyroid carcinoma via sequestering miR-2861. Exp Ther Med 2019;17:895-900. 
9 Du Y, Weng X-D, Wang L, et al. LncRNA XIST acts as a tumor suppressor in prostate cancer through sponging miR-23a to modulate RKIP expression. Oncotarget 2017;8:94358.

10 Xie F, Xiang X, Huang Q, et al. Reciprocal control of IncRNA-BCAT1 and $\beta$-catenin pathway reveals IncRNA-BCAT1 long non-coding RNA acts as a tumor suppressor in colorectal cancer. Oncotarget 2017;8:23628.

11 Qian H, Chen L, Huang J, et al. The IncRNA MIR4435-2HG promotes lung cancer progression by activating $\beta$-catenin signalling. J Mol Med 2018;96:753-64.

12 Meng Q, Ren M, Li Y, et al. LncRNA-RMRP acts as an oncogene in lung cancer. PLoS One 2016;11:e0164845.

13 Bai X, Wang W, Zhao P, et al. LncRNA CRNDE acts as an oncogene in cervical cancer through sponging miR-183 to regulate CCNB1 expression. Carcinogenesis 2020;41:111-21.

14 Zhang Y, Zhang D, Lv J, et al. LncRNA SNHG15 acts as an oncogene in prostate cancer by regulating miR-338-3p/FKBP1A axis. Gene 2019;705:44-50.

15 Wang Y, Fu J, Wang Z, et al. Screening key IncRNAs for human lung adenocarcinoma based on machine learning and weighted gene co-expression network analysis. Cancer Biomarkers 2019;25:31324.

16 Santander S, Cebrián C, Esquivias $\mathrm{P}$, et al. Cyclooxygenase inhibitors decrease the growth and induce regression of human esophageal adenocarcinoma xenografts in nude mice. Int J Oncol 2012;40:527-34.

17 Wan Y, Yang Z-Q. LncRNA NEAT1 affects inflammatory response by targeting miR-129-5p and regulating Notch signaling pathway in epilepsy. Cell Cycle 2020;19:419-31.

18 Cai C, Zhi Y, Wang K, et al. CircHIPK3 overexpression accelerates the proliferation and invasion of prostate cancer cells through regulating miRNA-338-3p. Onco Targets Ther 2019;12:3363.

19 Cui J, Li W, Liu G, et al. A novel circular RNA, hsa_circ_0043278, acts as a potential biomarker and promotes non-small cell lung cancer cell proliferation and migration by regulating miR-520f. Artif cells, nanomedicine, Biotechnol 2019;47:810-21.

20 Acha-Sagredo A, Uko B, Pantazi P, et al. Long non-coding RNA dysregulation is a frequent event in nonsmall cell lung carcinoma pathogenesis. Br J Cancer 2020;122:1050-8.

21 Salavaty A, Rezvani Z, Najafi A. Survival analysis and functional annotation of long non-coding RNAs in lung adenocarcinoma. J Cell Mol Med 2019;23:5600-17.

22 Chen S, Wang M, Yang $\mathrm{H}$, et al. LncRNA TUG1 sponges microRNA-9 to promote neurons apoptosis by up-regulated Bcl2l11 under ischemia. Biochem Biophys Res Commun 2017;485:167-73. 
23 Liang W-C, Fu W-M, Wong C-W, et al. The IncRNA H19 promotes epithelial to mesenchymal transition by functioning as miRNA sponges in colorectal cancer. Oncotarget 2015;6:22513.

$24 \mathrm{Liz} \mathrm{J}$, Esteller M. IncRNAs and microRNAs with a role in cancer development. Biochim Biophys Acta (BBA)-Gene Regul Mech 2016;1859:169-76.

25 Luo L, Zhang J, Tang H, et al. LncRNA SNORD3A specifically sensitizes breast cancer cells to 5-FU by sponging miR-185-5p to enhance UMPS expression. Cell Death Dis 2020;11:1-12.

26 Zhang H, Lu W. LncRNA SNHG12 regulates gastric cancer progression by acting as a molecular sponge of miR-320. Mol Med Rep 2018;17:2743-9.

27 Tao F, Tian X, Lu M, et al. A novel IncRNA, Lnc-OC1, promotes ovarian cancer cell proliferation and migration by sponging miR-34a and miR-34c. J Genet Genomics 2018;45:137-45.

28 Zhang Z, Cheng J, Wu Y, et al. LncRNA HOTAIR controls the expression of Rab22a by sponging miR373 in ovarian cancer. Mol Med Rep 2016;14:2465-72.

$29 \mathrm{Xu} \mathrm{M}$, Chen X, Lin K, et al. IncRNA SNHG6 regulates EZH2 expression by sponging miR-26a/b and miR214 in colorectal cancer. $J$ Hematol Oncol 2019;12:3.

30 Bao Y, Li K, Guo Y, et al. Tumor suppressor PRSS8 targets Sphk1/S1P/Stat3/Akt signaling in colorectal cancer. Oncotarget 2016;7:26780.

31 Bao Y, Guo Y, Yang Y, et al. PRSS8 suppresses colorectal carcinogenesis and metastasis. Oncogene 2019;38:497-517.

32 Chen L-M, Verity NJ, Chai KX. Loss of prostasin (PRSS8) in human bladder transitional cell carcinoma cell lines is associated with epithelial-mesenchymal transition (EMT). BMC Cancer 2009;9:377.

33 Tamir A, Gangadharan A, Balwani S, et al. The serine protease prostasin (PRSS8) is a potential biomarker for early detection of ovarian cancer. J Ovarian Res 2016;9:1-13.

$34 \mathrm{Ma} \mathrm{C}$, Ma W, Zhou N, et al. Protease serine S1 family member 8 (PRSS8) inhibits tumor growth in vitro and in vivo in human non-small cell lung cancer. Oncol Res Featur Preclin Clin Cancer Ther 2017;25:7817.

\section{Tables}

Due to technical limitations, table 1 is only available as a download in the Supplemental Files section.

\section{Figures}


Fig.1

A

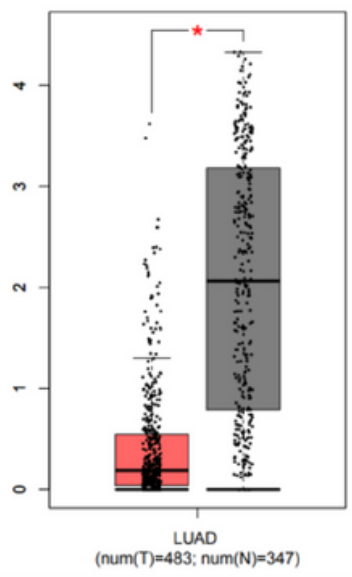

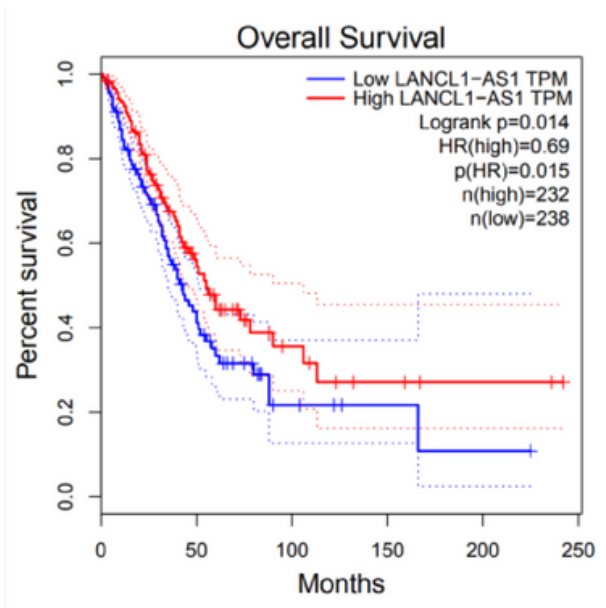

B

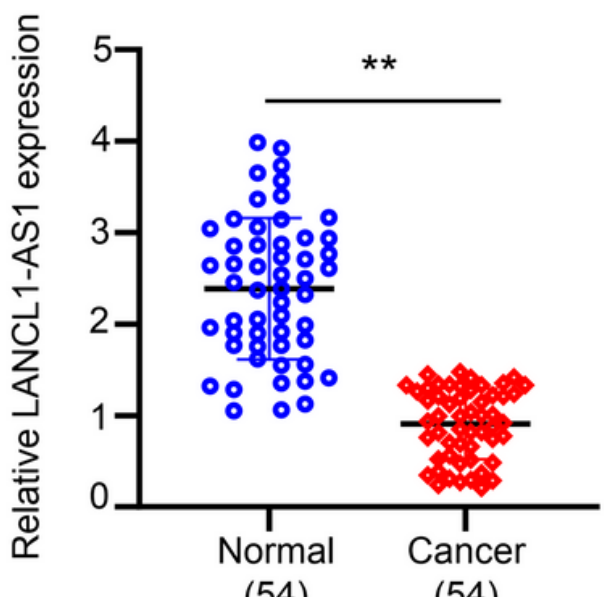

(54)

(54)
C

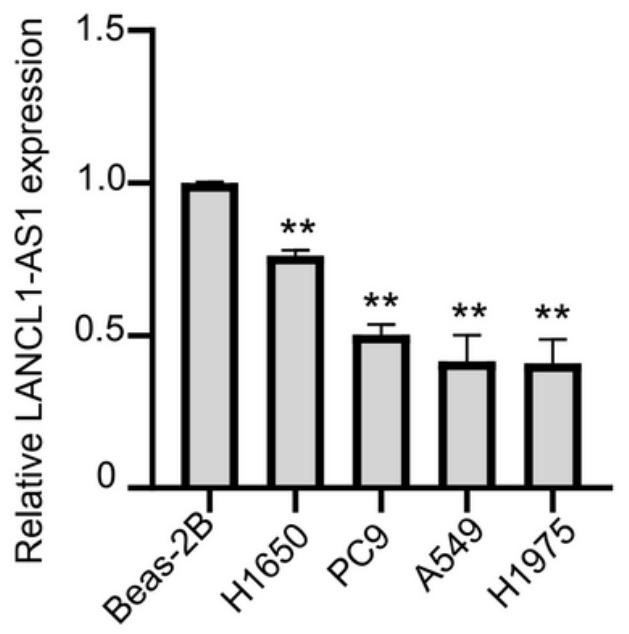

D

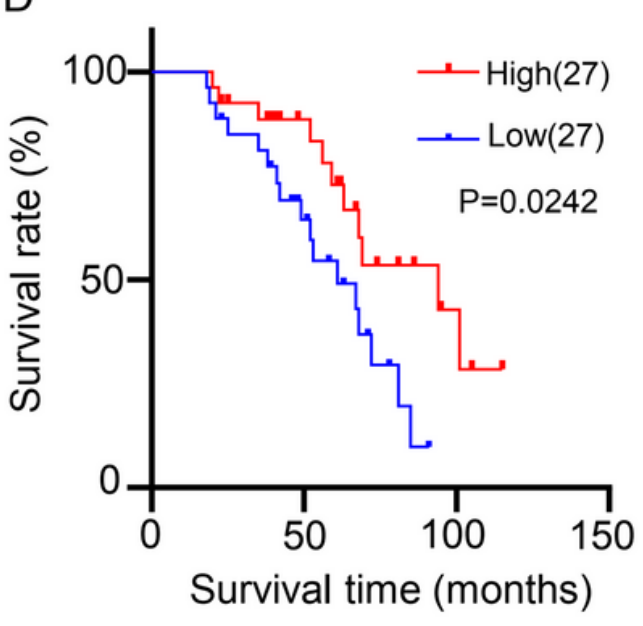

\section{Figure 1}

LncRNA LANCL1-AS1 is expressed at low levels in LUAD tissue and cell line. (A) TGCA online database analysis showed that LANCL1-AS1 expression decreased in LUAD that was associated with decreased survival. (B) The expression level of LANCL1-AS1 in 54 pairs of LUAD tissues and adjacent tissues was detected by qRT-PCR. (C) The expression level of LANCL1-AS1 in LUAD cell line (A549, H1975, H1650, and PC9) and normal lung epithelial cell line Beas-2B was detected by qRT-PCR. Kaplan-Meier survival curve after the median expression value of LANCL1-AS1 in LUAD tissue in Fig.1C was used as cut-off value and 54 LUAD were divided into high expression group $(n=27)$ and low expression group $(n=27)$. Statistical analysis has been described under the Materials and Methods section. ${ }^{*}=P<0.05, * *=P<0.01$ 
Fig.2
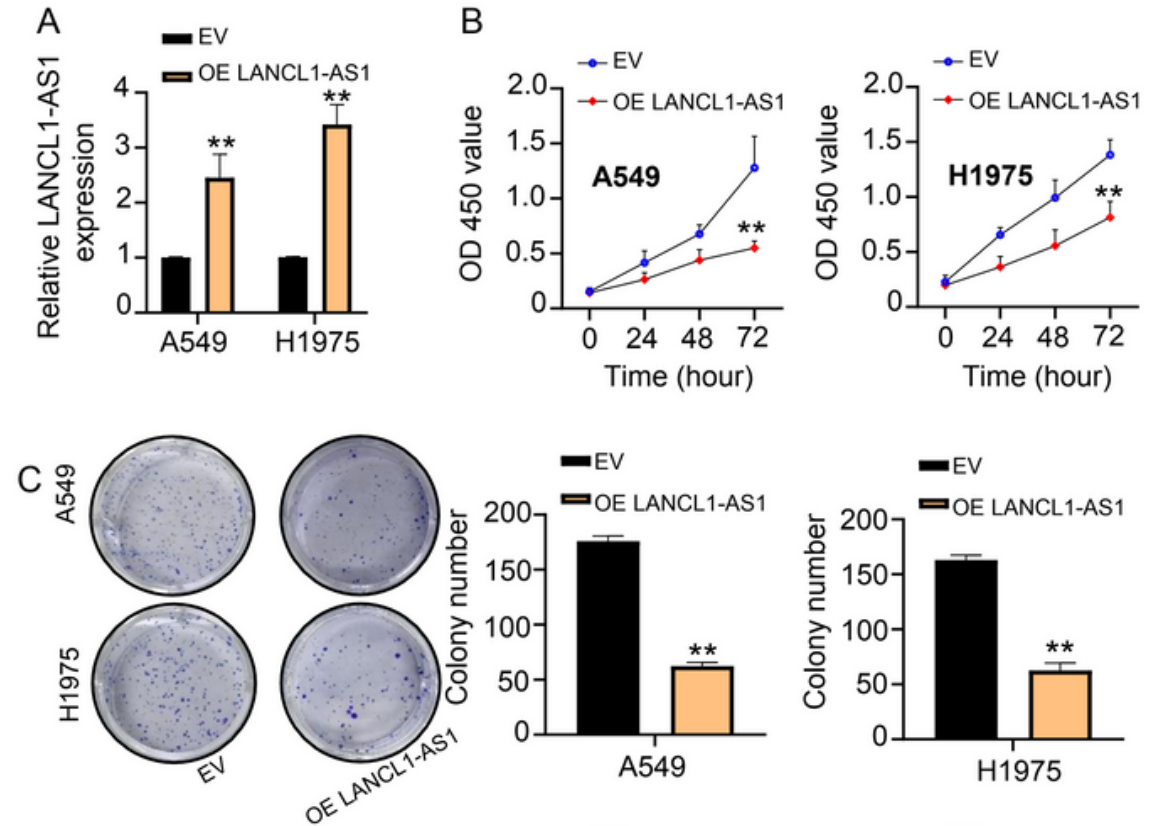

D
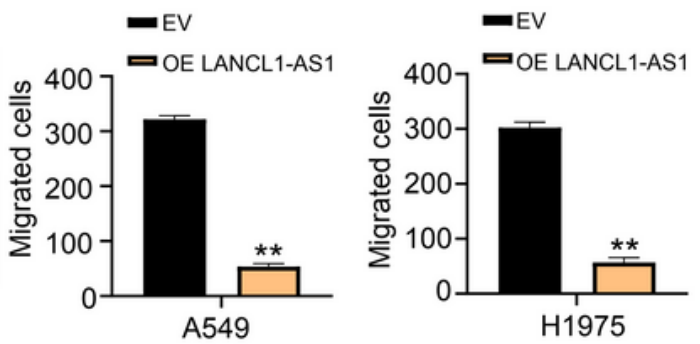

E
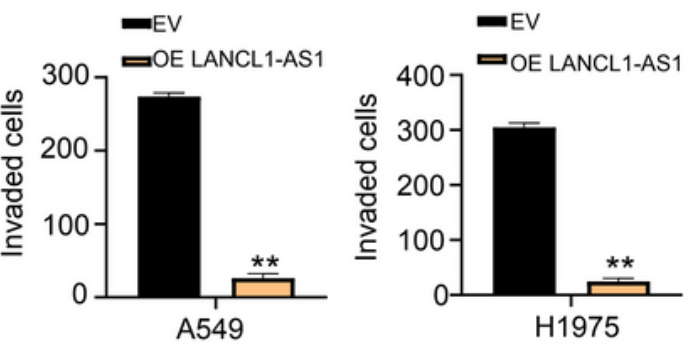

F
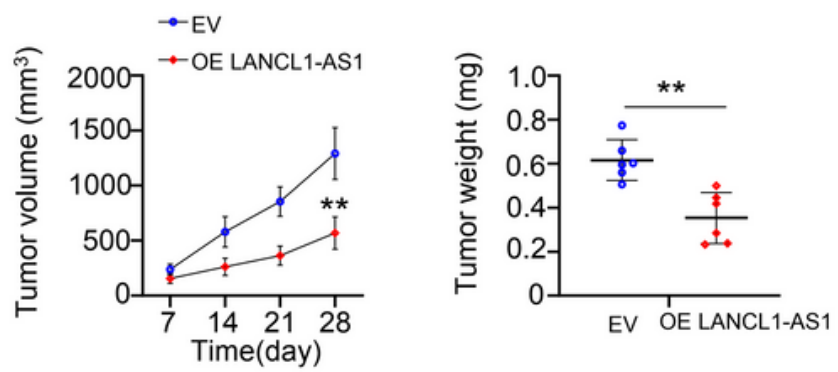

Figure 2

LANCL1-AS1 overexpression decreases the viability, proliferation, migration and invasion of LUAD cells. (A) LANCL1-AS1 was overexpressed in LUAD cell lines and the overexpression was confirmed by qRTPCR. (B) CCK8 assay to detect the absorbance values of A549 and H1975 cells in different groups. (C) Colony formation assay. (D, E) Transwell assay for the determination of the migration and invasion 
capacity of LUAD cells. (F) Tumor growth in nude mice in terms of volume and weight. Statistical analysis has been described under the Materials and Methods section. ${ }^{*}=P<0.05,{ }^{*}=P<0.01$

Fig.3

A

LANCL1-AS1 WT 5'-UAACAUGCAAAAAGGGGA--AGAGGACAGGA-3' miR-6748-3p

LANCL1-AS1 Mut 5'-UAACAUGCAAAAUCCGCU--UCACCUGUCCU-3'

C
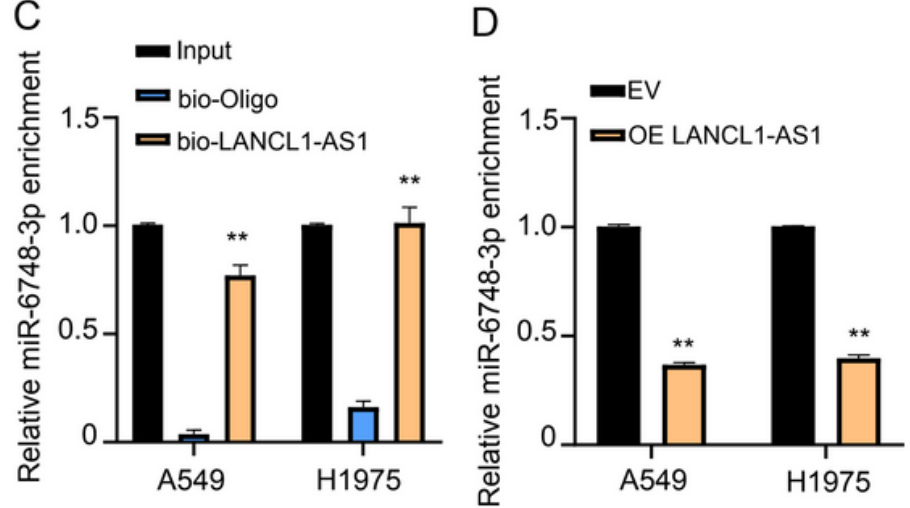

$\mathrm{B}$

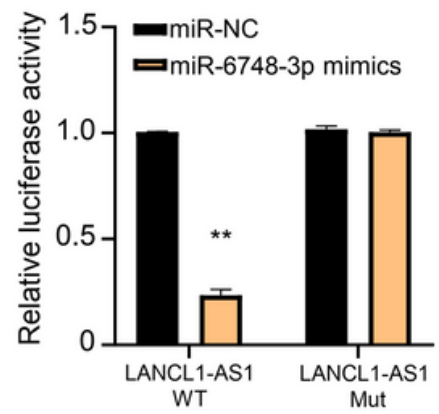

E

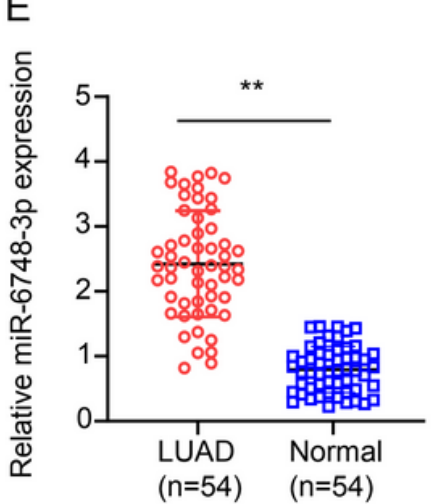

H1975

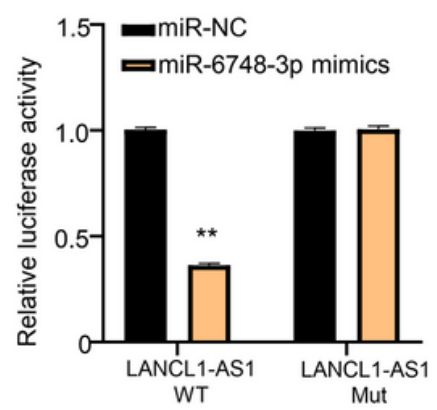

F

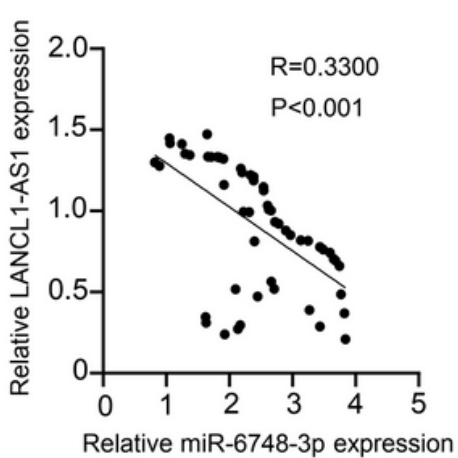

\section{Figure 3}

miR-6748a-3p is the target of LncRNA LANCL1-AS1. (A) miR-6748a-3p binding site on LANCL1-AS1 was predicted by LncBase online database. (B) Luciferase reporter gene experiments on in A549 and h1975 cells. (C) RNA pull down experiment with biotin-labeled LANCL1-AS1 probe. (D) Effect of LANCL1-AS1 over expression on the expression of miR-6748-3p. (E) The expression level of miR-6748a-3p in 54 pairs of LUAD tissues and normal tissues adjacent to cancer. (F) Pearson correlation coefficient was used to analyze the correlation between LANCL1-AS1 and miR-6748a-3p expression. Statistical analysis has been described under the Materials and Methods section. ${ }^{*}=\mathrm{P}<0.05,{ }^{*}=\mathrm{P}<0.01$ 
Fig.4

A

PRSS8 WT

5'-AUGCAUCACACUCAAGGACAGGA-3'

miR-6748-3p 3'-GACAUCCUCUGUCCCUGUCCU-5'

PRSS8 Mut 5'-AUGCAUCACACUCAACCUGUCCU-3'

C

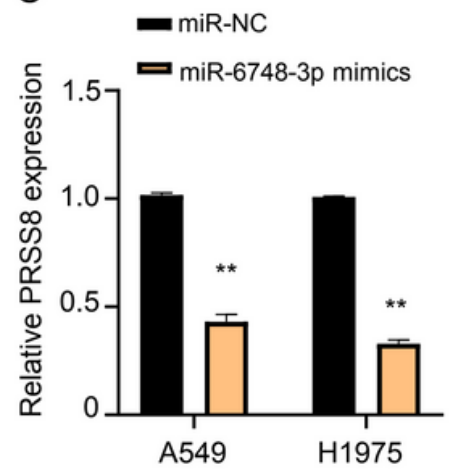

B
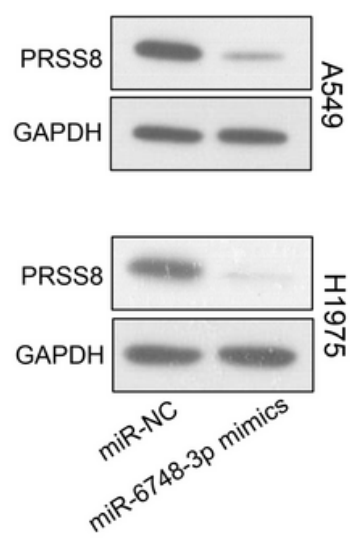

A549

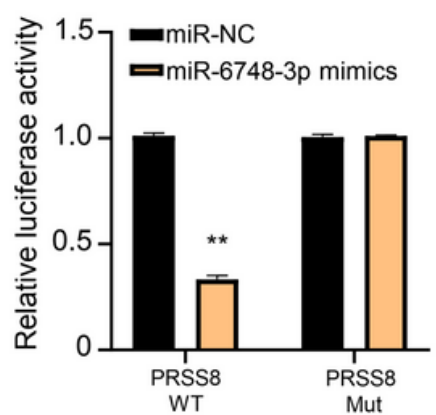

$E$

H1975

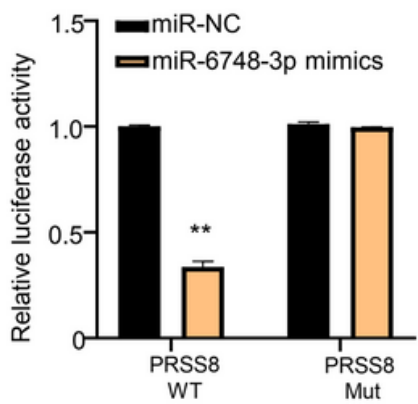

$\mathrm{F}$

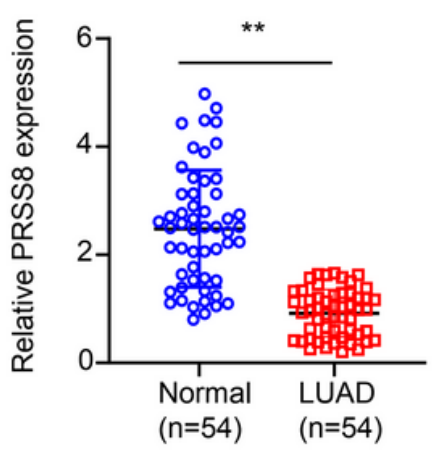

Figure 4

miR-6748a-3p targets PRSS8. (A) A: Targetscan database predicated that PRSS8 had the miR-6748a3pbinding sites. (B) Luciferase reporting experiments in A549 and h1975 cells respectively. (C, D) qRTPCR and western blots were used to detect the mRNA and protein expression level of PRSS8 mRNA in A549 and H1975 cells after overexpression of miR-6748a-3p. (E) Pearson correlation coefficient to analyze the correlation between PRSS8 and miR-6748a-3p expression. Statistical analysis has been described under the Materials and Methods section. ${ }^{*}=\mathrm{P}<0.05,{ }^{*}=\mathrm{P}<0.01$ 
Fig.5
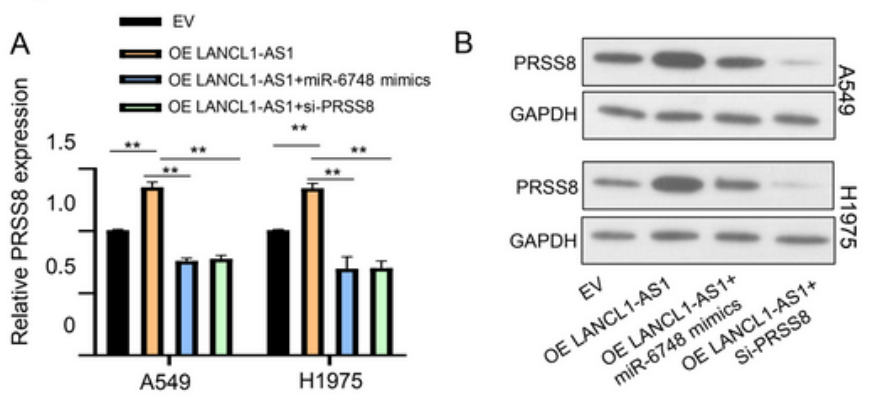

C

- EV

- EV

$\rightarrow$ OE LANCL1-AS1
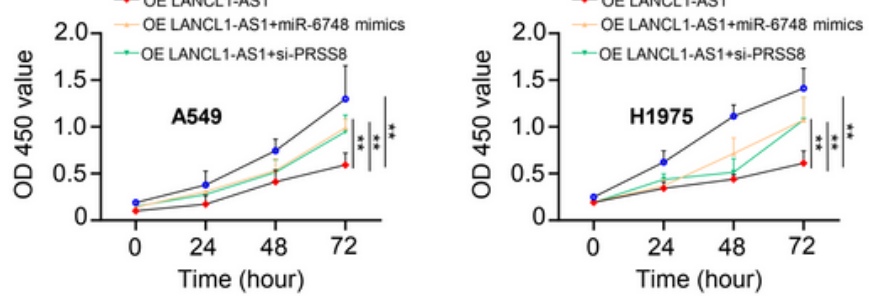

D
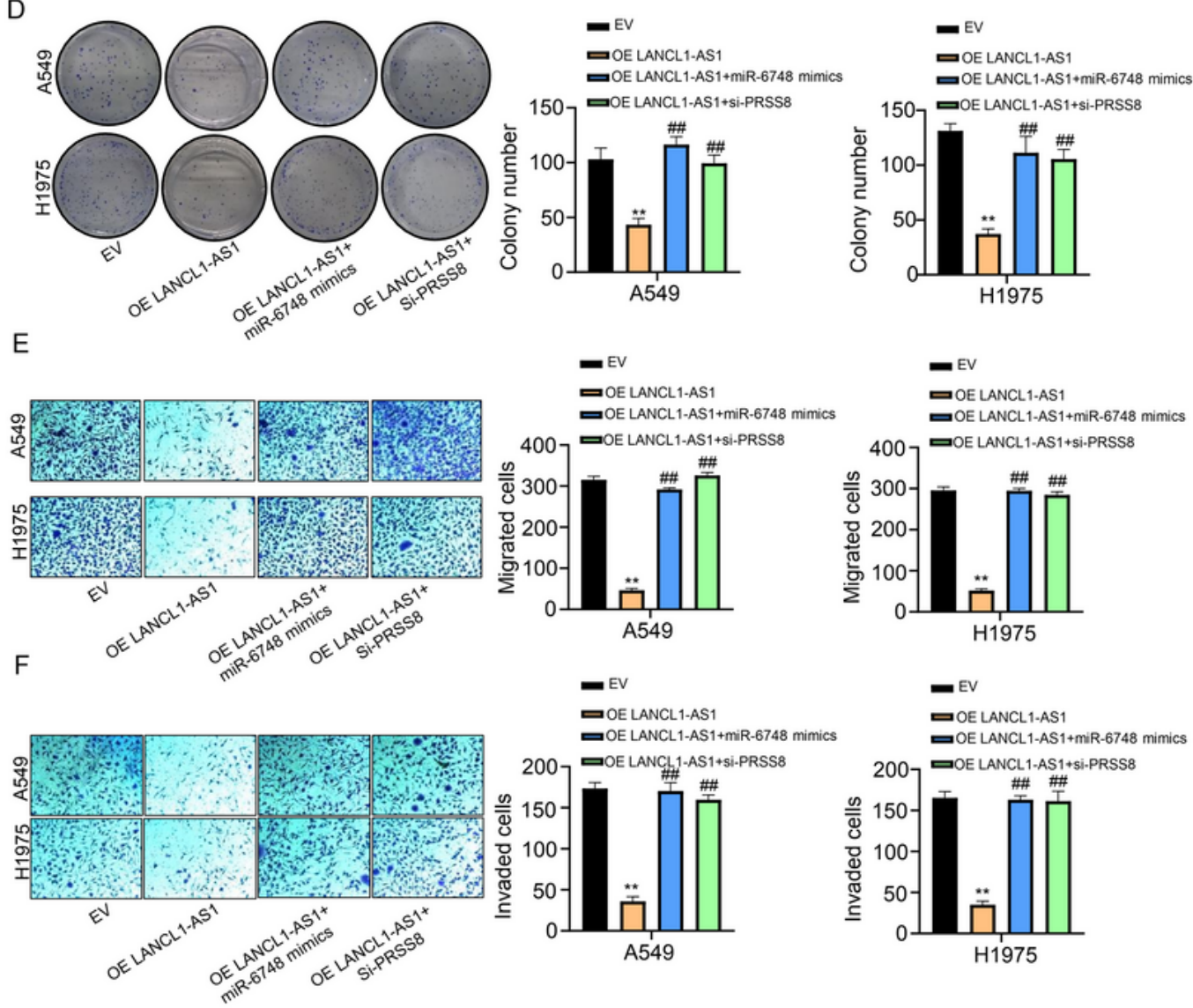

\section{Figure 5}

LANCL1-AS1 suppresses LUAD progression by sponging miR-6748a-3p and subsequent upregulation of PRSS8. (A, B) The qRT-PCR and western blot were used to detect mRNA and protein expression of PRSS8 in different groups (EV, OE LANCL1-AS1, OE LANCL1-AS1+miR-6748 mimics, OE LANCL1-AS1+si-PRSS8). (C) CCK-8 assay, (D) Colony formation assay, trasnwell assay for migration (E) and invasion (F) capacity of LUAD cells in different groups (EV, OE LANCL1-AS1, OE LANCL1-AS1+miR-6748 mimics, OE LANCL1- 
AS1+si-PRSS8). Statistical analysis has been described under the Materials and Methods section. ${ }^{*}=\mathrm{P}<$ $0.05, * *=\mathrm{P}<0.01$.

\section{Supplementary Files}

This is a list of supplementary files associated with this preprint. Click to download.

- Table1.docx

- SupportingmaterialWB.pptx

- AuthorChecklist.pdf 